\title{
Tooth whitening efficacy of pigmented rice gels containing carbamide peroxide
}

\author{
Adchareeya Kaewpinta ${ }^{1,2}$, Sakornat Khongkhunthian ${ }^{2,3}$, Pisaisit Chaijareenont ${ }^{2,4}$, \\ Siriporn Okonogi ${ }^{2,5, *}$
}

${ }^{1}$ Interdisciplinary Program in Nanoscience and Nanotechnology, The Graduate School, Chiang Mai University, Chiang Mai, Thailand;

${ }^{2}$ Research Center of Pharmaceutical Nanotechnology, Chiang Mai University, Chiang Mai, Thailand;

${ }^{3}$ Department of Restorative Dentistry and Periodontology, Faculty of Dentistry, Chiang Mai University, Chiang Mai, Thailand;

${ }^{4}$ Department of Prosthodontics, Faculty of Dentistry, Chiang Mai University, Chiang Mai, Thailand;

${ }^{5}$ Department of Pharmaceutical Sciences, Faculty of Pharmacy, Chiang Mai University, Chiang Mai, Thailand.

\begin{abstract}
Summary Carbamide peroxide (CP) is commonly used as a tooth whitening agent. However, efficacy of CP can be enhanced if it is in the suitable delivery system. In the present study, CP loaded in pigmented rice gels were developed and investigated for their physicochemical properties and tooth whitening efficacy. The modified pigmented rice of two varieties, Homnil (HN) and Doisket (DS) were prepared and used as a gelling agent. The outer appearance of the obtained rice gels containing $10 \% \mathrm{CP}$ (CP-HN and CP-DS, respectively) were transparent and homogeneous texture. The $\mathrm{pH}$ of both rice gel bases was neutral but became slightly acidic after incorporating with $\mathrm{CP}$. The adhesive property of HN gel was significantly higher than DS gels. In vitro drug release profile exhibited that the release of $\mathrm{CP}$ from $\mathrm{CP}$ DS was significantly higher than CP-HN and the commercial gel (CP-CG), respectively. In vitro tooth whitening efficacy in 45 normal teeth revealed that the tooth whitening efficacy of the gels was time dependent. At the end of the treatment, CP-HN showed significantly higher tooth whitening efficacy than CP-DS and CP-CG, respectively. It is concluded that the physicochemical properties, particularly the adhesive and dissolution properties, play an important role in the tooth whitening efficacy of the CP gels.
\end{abstract}

Keywords: Homnil rice, Doisket rice, colored rice gels, tooth whitening, drug release

\section{Introduction}

Abnormal tooth's color or tooth discoloration is the stains on the teeth that are yellow, gray, chalky white, or brownish in color. It also refers to spots, blemishes, or lines on tooth surfaces (1). Stains on the teeth has many different causes; bacterial, food, aging, smoking, and some drugs such as fluoride, tetracycline, and doxycycline (2). Drinking colored beverage like coffee, tea, and red wine can also affect to the tooth color (3). All of these reasons cause a reduction in the brilliance of the enamel and dentin. Tooth discoloration affects

\footnotetext{
*Address correspondence to:

Dr. Siriporn Okonogi, Department of Pharmaceutical Sciences, Faculty of Pharmacy, Chiang Mai University, Chiang Mai 50200, Thailand.

E-mail: okng2000@gmail.com
}

physical appearance, beauty, and self-confidence (4). In adolescence, tooth discoloration can cause an effect on their psychosocial development $(5,6)$.

Tooth whitening agents commonly used are hydrogen peroxide and carbamide peroxide (CP) (7). $\mathrm{CP}$ is a white crystal powder and a potent water-soluble agent. It acts as a strong oxidizing agent through the formation of free radicals, reactive oxygen molecules, and hydrogen peroxide anions. These reactive molecules attack the long-chained, dark-colored chromophore molecules and then split them into smaller, less colored and more diffusible molecules $(7,8)$. $\mathrm{CP}$ is a popular tooth whitening ingredient because of its effectiveness and quite safe $(9,10)$.

The most common formulation of tooth whitening products is gels, due to their preferable in terms of patient compliance, comfortable and easy to applyon the tray that used for tooth whitening (11). Gels have 
generally long chain molecular structure composed of many single molecules of monomers bonded to each other which called polymer. Gelling agents can be categorized into two groups: synthetic polymers and natural polymers. Nowadays the interesting in natural polymers produced from natural raw materials is increase because of environmental benefits (12). Rice grain is mainly composed of carbohydrate, which is also a natural polymer. The main components of rice carbohydrate are amylose and amylopectin. Amylose is a linear polymer chain and amylopectin is a branch polymer chain $(13,14)$. The structure of starch in rice grain can be modified in order to create a preferable gelling agent $(15,16)$. There has been reported the application of modified rice as a gelling agent in pharmaceutical fields especially using as the drug carrier gels in the buccal cavity (17) due to their biodegradability $(18,19)$. Rice can be categorized into two groups based on the color of rice grains, pigmented and non-pigmented groups. The pigmented rice varieties have been received increasing interest currently due to their high bioactive compounds, such as phenolics, tocols, sterol derivatives which have high antioxidant, anti-inflammatory, and other health benefits $(20,21)$. Therefore, the pigmented rice was selected to use in the present study. The physicochemical properties, such as $\mathrm{pH}$, adhesive, and drug release properties of the pigmented rice gels obtained from two varieties were compared and the tooth whitening efficacy of the obtained CP loaded rice gels were investigated.

\section{Materials and Methods}

\subsection{Materials}

Thai pigmented rice grain varieties; Homnil (HN) and Doisket (DS), were obtained from local market in Chiang Mai province, Thailand. CP, silver nitrate, triphenylphosphine (TPP), and monochloroacetic acid were from Sigma chemical Co. (St. Louis, MO, USA). Dichloromethane, methanol, and glacial acetic acid were from RCI Lab-scan Co., Ltd. (Bangkok, Thailand). A commercial gel (CP-CG) containing the same concentration of CP was obtained from Ultradent Product Inc. (Salt Lake City, UT, USA). All other chemicals and solvents were of analytical grade or the highest grade available.

\subsection{Preparation of $C P$ rice gels}

\subsubsection{Preparation of modified rice powder}

Raw pigmented rice powder samples of HN and DS prepared by wet milling method (22) were modified using etherification method previously described (17) with some modification. Briefly, 50\% (w/w) sodium hydroxide aqueous solution was mixed with methanol at a ratio of 1:4 (w/w) in a 100-mL three necked round- bottom flask. The raw rice powder was added and stirred at room temperature until homogenous slurry was obtained. After that proper amount of monochloroacetic acid was added. The temperature of the mixture was controlled at $50 \pm 5^{\circ} \mathrm{C}$ for $3 \mathrm{~h}$ with continuous stirring. At the end of the reaction, the mixture was neutralized to $\mathrm{pH}$ 7.0. The solid phase was collected and washed by $80 \%$ (w/w) methanol until the filtrate testing for chloride by using silver nitrate test was negative. The obtained slurry was dried at $50^{\circ} \mathrm{C}$ for $48 \mathrm{~h}$. The obtained dried solid was pulverized and passed through the 80 -mesh sieve. The modified rice powder after passing the 80 -mesh sieve was kept in a desiccator for further use.

\subsubsection{Preparation of rice gel bases and CP rice gels}

Modified HN and DS powder samples were dispersed in DI water and heated to $90^{\circ} \mathrm{C}$ for $1 \mathrm{~h}$ with gentle stirring to obtain homogenous gels without air bubble. $\mathrm{CP}$ was gradually incorporated into the prepared gel bases to obtain a final concentration of $10 \% \mathrm{HN}$ gels containing 10\% CP (CP-HN) and DS gels containing $10 \% \mathrm{CP}(\mathrm{CP}-\mathrm{DS})$ were stored at $4{ }^{\circ} \mathrm{C}$ until further use.

\subsection{Determination of $p H$}

The $\mathrm{pH}$ of the gel bases and the $\mathrm{CP}$ rice gels, $\mathrm{CP}-\mathrm{HN}$ and CP-DS, as well as the commercial gel, CP-CG was determined at room temperature using a $\mathrm{pH}$ meter (Compact pH meter pH 22 Laqua twin, Horiba, Kyoto, Japan). The $\mathrm{pH}$ meter was standardized using $\mathrm{pH} 4$ and pH 7 buffers before use.

\subsection{Adhesive property study}

The adhesive property of the rice gel bases, CP rice gels, and CP-CG was investigated using the method previously described (23) with some modification. The exact amount of $1 \mathrm{~g}$ of gels was applied on a $2 \mathrm{~cm} \times$ $2 \mathrm{~cm}$ area of a porcine intestinal mucosal membrane. The membrane was freshly cut and fixed on the internal side of the beaker. The beaker was filled with $800 \mathrm{~mL}$ artificial saliva at $37 \pm 1^{\circ} \mathrm{C}$. The water was constantly stirred at $150 \mathrm{rpm}$. The time of the gels detachment from the membrane was recorded.

\subsection{In vitro drug release property}

In vitro drug release study was performed using dialysis bag with a molecular weight cut-off at 12,000 daltons (Cellu Sep ${ }^{\circledR}$ T4 regenerated cellulose tubular membrane, Membrane filtration products Inc., Seguin, TX, USA). The dialysis bag was activated by using the company method before starting the experiment. Briefly, the dialysis bag was soaked in $100 \mathrm{~mL}$ of distilled water and heated at $100^{\circ} \mathrm{C}$ for $30 \mathrm{~min}$, then kept overnight at $4^{\circ} \mathrm{C}$. The amount of $1 \mathrm{~g}$ of gel sample was placed in the 
activated dialysis bag without air bubbles. The bag was tightly closed and immersed into the $50-\mathrm{mL}$ medium (artificial saliva). The medium was maintained at $37 \pm$ $1^{\circ} \mathrm{C}$ under constant stirring of $100 \mathrm{rpm}$. The samples were collected after $5,10,15,20,30,40,50$, and 60 min. The volume of the collected sample was replaced with the fresh receptor medium. The amount of CP released was determined using high performance liquid chromatography (HPLC) (Hewlett Packard series 1100, Agilent technologies, Santa Clara, CA, USA) according to the previous method (24) with some modification. Briefly, 1,000 mL of 0.1 M TPP was added to $1,000 \mathrm{~mL}$ of the samples and constantly stirred for $2 \mathrm{~h}$ with light protection. The condition of HPLC was as follows; a reversed phase column Hypersil ODS $(4.6 \times 250 \mathrm{~mm}$, Agilent technologies) was used as a stationary phase, UV detection was at $225 \mathrm{~nm}$, a mobile phase with flow rate of $1.0 \mathrm{~mL} / \mathrm{min}$ consisted of acetonitrile and water gradient. At starting time, the mobile phase was $50 \%$ acetonitrile and $50 \%$ water, then at $6.5 \mathrm{~min}$ of running time it was changed to $100 \%$ acetonitrile. At $10 \mathrm{~min}$ of running time the mobile phase was changed to $50 \%$ acetonitrile with $50 \%$ water, and this mixture was continued running until the retention time was completed at $25 \mathrm{~min}$. The injection volume was 10 $\mu \mathrm{L}$ and the running temperature was $25 \pm 0.2^{\circ} \mathrm{C}$. A calibration curve was prepared using $\mathrm{CP}$ solution at concentration range of $50-200 \mu \mathrm{g} / \mathrm{mL}$. The standard curve gave a linear response with correlation coefficient $\left(r^{2}\right)$ of 0.9997 .

\subsection{In vitro tooth whitening efficacy study}

\subsubsection{Tooth preparation}

Forty-five normal teeth were collected by dentists from normal volunteers of Chiang Mai University (CMU). This study was approved by the Human Experimentation Committee, Faculty of Dentistry, Chiang Mai University (No. 21/255). Normal teeth without hypoplastic defects were cleaned. The imperfections of the teeth were removed then stored at $4^{\circ} \mathrm{C}$ in saturated $0.1 \%$ thymol solution until use.

\subsubsection{Evaluation of tooth whitening efficacy}

Color of the teeth was measured by using colorimeter (Fru WR10 portable precision colorimeter, Shenzhen wave optoelectronics technology Co., Ltd, Shenzhen, China) with color values recorded from $3 \mathrm{~mm}$ circular centers of tooth surface. The color values were calculated using the Commission international de l'Eclairage (International commission on illumination: CIE) b* (yellow-blue) scales $(25,26)$.

The teeth were randomly allocated into 5 groups, including the test groups of CP-HN and CP-DS, the positive control group of $\mathrm{CP}-\mathrm{CG}$, and the gel bases groups of $\mathrm{HN}$ and DS as the negative control groups. Regimen of the treatment was $1 \mathrm{~h} /$ day, $4 \mathrm{~h} /$ day and $8 \mathrm{~h} /$ day, duration of the study was 7 days. Each day, the exact amount of $0.1 \mathrm{~mL}$ samples were placed on enamel surface surrounded with $0.5 \mathrm{~mL}$ artificial saliva. The samples were kept in a close container with the controlled relative humidity of $100 \%$ and temperature of $25 \pm 0.2^{\circ} \mathrm{C}$. After testing time was completed, the teeth samples were removed and washed by deionized water. Subsequently, the color was measured and calculated for the whitening efficacy of the gels.

\subsection{Statistical analysis}

Descriptive statistics for continuous variables were calculated and reported as a mean \pm standard deviation (SD). Data were analyzed using a one-way analysis of variance (ANOVA) and Duncan's multiple range test ( $p$ $<0.05$ ) using SPSS statistic software version 22.

\section{Results}

\subsection{Outer appearance and $\mathrm{pH}$ of the gels}

The obtained CP pigmented rice gels showed homogenous textures with moderate viscosity. Phase separation and drug precipitation were not found in all gels. The pH values of the gel bases was slightly different from the CP loading gels as shown in Table 1. The $\mathrm{pH}$ of rice gels bases was nearly neutral. After CP was incorporated, the $\mathrm{pH}$ of the rice gels was decreased.

\subsection{Adhesive property of the gels}

The adhesive property of the gels was different as presented in Table 1. Comparison between two pigmented rice gel bases, HN gel base showed longer detachment time than DS gel base indicating that $\mathrm{HN}$ has higher adhesive property than DS gel base. After CP was loaded, the adhesive property of the gels was slightly decreased. Considering the detachment time of gels containing $\mathrm{CP}, \mathrm{CP}-\mathrm{HN}$ had the highest detachment time followed by CP-CG and CP-DS.

\subsection{In vitro drug release property}

The determination of $\mathrm{CP}$ released from the gels was performed using HPLC method based on an oxidation

Table 1. pH values and adhesive property of the gels

\begin{tabular}{lcc}
\hline Gels & $\mathrm{pH}$ & Adhesion, Detachment time (min) \\
\hline HN & $7.83 \pm 0.03$ & $64 \pm 1$ \\
DS & $7.64 \pm 0.02$ & $47 \pm 3$ \\
CP-HN & $6.62 \pm 0.02$ & $56 \pm 3$ \\
CP-DS & $6.16 \pm 0.01$ & $42 \pm 2$ \\
CP-CG & $6.51 \pm 0.02$ & $49 \pm 2$ \\
\hline
\end{tabular}




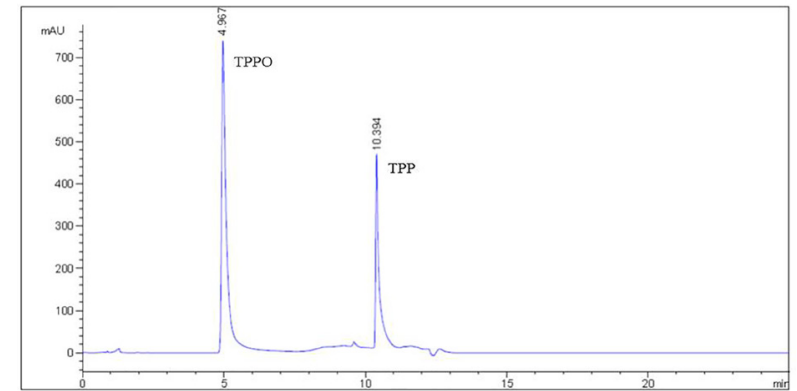

Figure 1. HPLC chromatograms of TPPO and TPP.

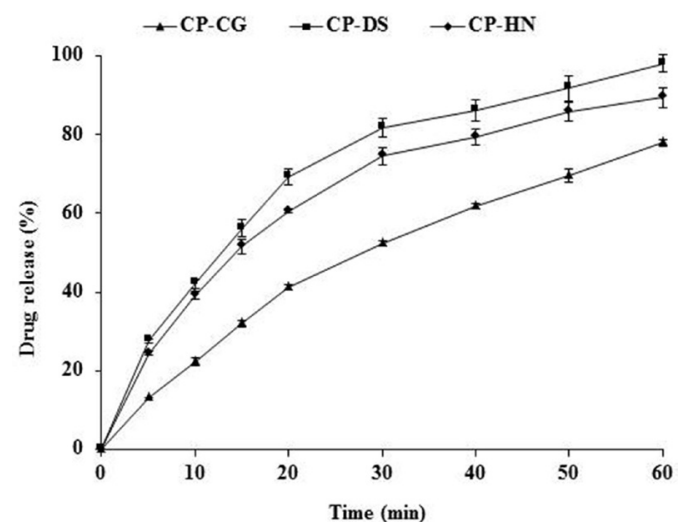

Figure 2. Release profiles of CP from CP-HN, CP-DS, and CP-CG.

of TPP into triphenylphosphine oxide (TPPO) (27). TPP and TPPO presented the HPLC chromatogram peaks at different retention times as presented in Figure 1. Determination of CP was made by external quantification using TPPO peaks area.

In vitro drug release of the two pigmented rice gels containing CP and CP-CG was presented in Figure 2. The results showed that the fastest drug release was obtained from CP-DS followed by CP-HN and CP$\mathrm{CG}$, respectively. During the release time, both $\mathrm{CP}$ rice gels demonstrated faster drug release than CP-CG. The amounts of the drug release from CP-DS, CP-HN and CP-CG after $60 \mathrm{~min}$ were $98.71 \pm 2.11 \%, 89.48 \pm$ $2.52 \%$, and $77.90 \pm 0.84 \%$ respectively.

\subsection{In vitro whitening efficacy}

Color of the teeth was measured by light reflection method using a colorimeter. This measurement reflects three color parameters; $\mathrm{L}^{*}, \mathrm{a}^{*}$, and $\mathrm{b}^{*}$. The parameter $\mathrm{L}^{*}$ refers to the lightness $(\mathrm{L})$, and ranges from black $(\mathrm{L}=0)$ to white $(\mathrm{L}=100)$. The parameter $\mathrm{a}^{*}$ refers to red-green color, a positive $\mathrm{a}^{*}$ refers to red and a negative $\mathrm{a}^{*}$ refers to green. The parameter $b^{*}$ refers to yellow-blue color, a positive $b^{*}$ indicates yellow and a negative $b^{*}$ indicates blue. The $\mathrm{L}^{*} \mathrm{a}^{*} \mathrm{~b}^{*}$ values were calculated for color changing by using CIE $\mathrm{L}^{*} \mathrm{a} * \mathrm{~b} *$ system as the following equation; $\Delta \mathrm{E}=\left[\left(\Delta \mathrm{L}^{*}\right)^{2}+\left(\Delta \mathrm{a}^{*}\right)^{2}+\left(\Delta \mathrm{b}^{*}\right)^{2}\right]^{1 / 2}(25,26)$. The $\Delta \mathrm{E}$ values are often used in tooth whitening studies in order to indicate the perceptible tooth color changes after

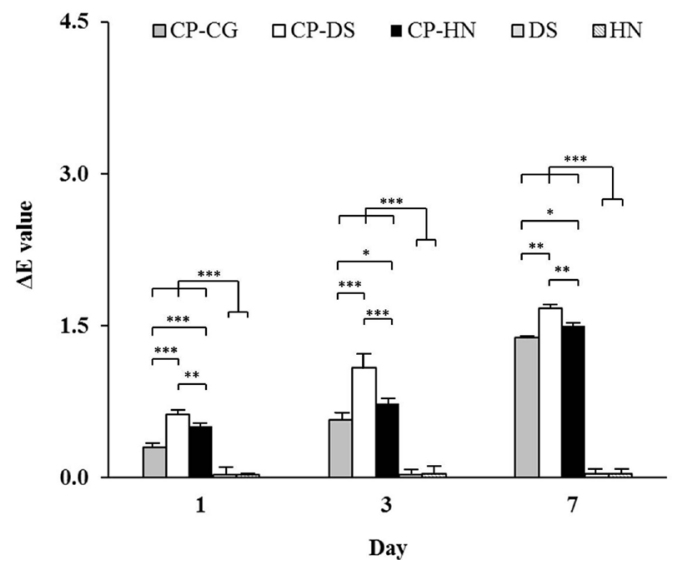

Figure 3. $\Delta \mathbf{E}$ value progression of 1-h regimen, values are given as means \pm SD. ${ }^{*} p<0.05,{ }^{* *} p<0.01$, and ${ }^{* * *} p<0.001$.

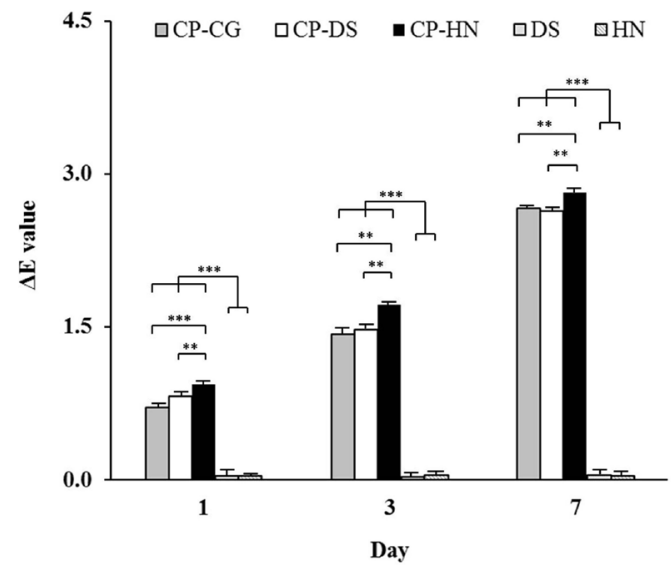

Figure 4. $\Delta \mathbf{E}$ value progression of 4-h regimen, values are given as means $\pm \mathbf{S D} .{ }^{* *} p<0.01$, and ${ }^{* * *} p<0.001$.

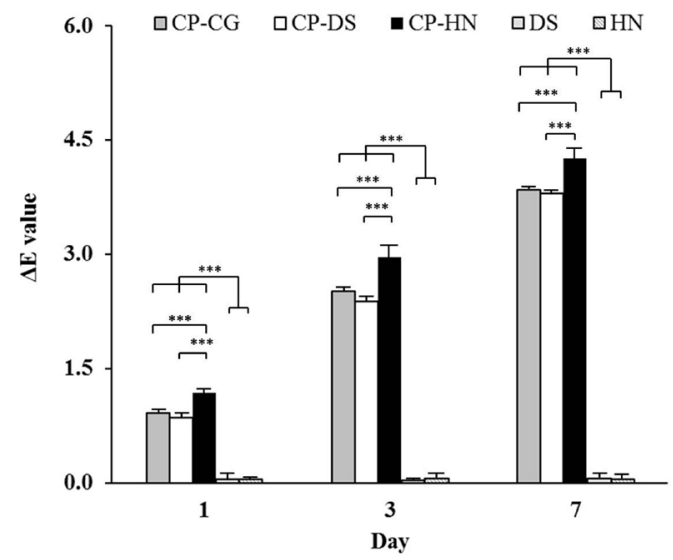

Figure 5. $\Delta \mathbf{E}$ value progression of 8 -h regimen, values are given as means \pm SD. $* * * p<0.001$.

treatment.

The $\Delta \mathrm{E}$ value progression at the different evaluation periods was presented in Figures 3-5. Comparing $\Delta \mathrm{E}$ values of all groups, it was demonstrated that all treatment groups; CP-HN, CP-DS and CP-CG, showed significantly higher $\Delta \mathrm{E}$ values than the negative control groups $(p<0.05)$. It was also visually observed that at day 7 , the tooth color of the treatment groups was 
whiter than that the tooth color at day 0 . At the end of the treatment, the $\Delta \mathrm{E}$ value of the treatment groups demonstrated significant tooth color improvement from day 0 , day 1 and day $3(p<0.05)$. It was also found that 8 -h regimen significantly improved tooth color when compared to 1 -h and 4 -h regimens $(p<0.05)$.

The $\Delta \mathrm{E}$ value of $1-\mathrm{h}$ regimen as shown in Figure 3 demonstrated that CP-DS group had the highest $\Delta \mathrm{E}$ value followed by CP-HN group and CP-CG group, the mean $\Delta \mathrm{E}$ value of day 7 was $1.67 \pm 0.04,1.50 \pm 0.03$, and 1.38 \pm 0.02 respectively. However the results of 4 -h regimen as shown in Figure 4 demonstrated that $\Delta \mathrm{E}$ value of $\mathrm{CP}$ $\mathrm{HN}$ was the highest. At day 7 , the mean $\triangle \mathrm{E}$ value of $\mathrm{CP}$ $\mathrm{HN}$ was $2.82 \pm 0.06$. CP-CG and CP-DS showed similar efficacy with the mean $\Delta \mathrm{E}$ values of $2.66 \pm 0.03$ and $2.64 \pm 0.04$, respectively. Similar results were found at 8-h regimen as shown in Figure 5, CP-HN showed the highest mean $\triangle \mathrm{E}$ value followed by $\mathrm{CP}-\mathrm{CG}$ and $\mathrm{CP}-\mathrm{DS}$ with the mean $\Delta \mathrm{E}$ values of $4.26 \pm 0.09,3.84 \pm 0.12$, and $3.79 \pm 0.07$ respectively. The results indicated that at the 1-h regimen, CP-DS was the most effective gels whereas at the 4-h and 8-h regimen $\mathrm{CP}-\mathrm{HN}$ was the most effective gels.

\section{Discussion}

According to the high hydrophilicity of the modified rice obtained from carboxymethylated etherification (28), preferable hydrophilic CP-HN gels and CP-DS gels can be successfully prepared. Both $\mathrm{CP}-\mathrm{HN}$ and $\mathrm{CP}-$ DS gels showed desirable transparency. CP can dissolve easily in water therefore the obtained $\mathrm{CP}$ rice gels shown homogenous texture without phase separation or drug precipitation. The $\mathrm{pH}$ of both rice gel bases was neutral but slightly decreased after $\mathrm{CP}$ was incorporated. Our results are in agreement with the work previously reported that the gels contained $\mathrm{CP}$ was slightly acidic (29). Therefore CP plays a role in the $\mathrm{pH}$ of the gels.

It has been known that adhesion is one of the most important properties in the pharmaceutical formulation in order to enhance localized drug delivery (30). Especially for tooth whitening formulations, a very high adhesive gels is preferable. It was reported that the enhancement of drug delivery through tooth surface and enamel could be successfully achieved by sufficient adhesive strength of formulations and teeth (31). Tooth whitening gels require high adhesive property to stay on the tooth surface to deliver maximum quantity of CP. The gels should not flow out from the tray to gingiva while using. High adhesion also helps to reduce side effect caused from peroxide damaged surrounding tissue $(32,33)$. The results from the present study showed that the adhesive property of HN gels was higher than that of DS gels. Our result is in correspondence with the results that have been previously reported $(15,19)$. This confirms that HN gels has higher adhesive property than DS gels. CP-HN and CP-DS showed similar adhesive property related to their respective gel bases. Moreover it was visually observed that $\mathrm{HN}$ and $\mathrm{CP}-\mathrm{HN}$ strongly attached to the membrane and slowly dissolved to the medium. DS and CP-DS showed easier dispatched from the membrane. This might be due to the different compositions of both rice. The positive control CP-CG was swelled and ruptured to small pieces then detached from the membrane. This result shows the low adhesive property of the commercial gels.

The results in tooth whitening efficacy of $\mathrm{CP}$ pigmented rice gels in human teeth indicate that the whitening efficacy of the gels is time dependent. The efficacy of 8-h regimen was significantly higher than that of $4-\mathrm{h}$ and $1-\mathrm{h}$ regimens, respectively and the results of 7-day treatment is obviously better than 3-day and 1-day treatments, respectively. This result is in correspondence with the previous $(34,35)$. Moreover, the results in the present study show that the adhesive and drug release properties of the gels play an important role in the tooth whitening efficacy. CP-DS gels showed the fastest drug release property compared to the other gels. At the 1-h regimen, CP-DS showed the highest tooth whitening efficacy. This result was in correspondence with in vitro release results. However at 4-h and 8-h regimens, CP-HN shows significantly higher effective tooth whitening efficacy than CPDS and CP-CG. This result indicates that the adhesive property of the gels influences the tooth whitening efficacy in these regimens. CP-HN gel has the highest adhesive property among the test gels and could retain at the tooth surface area for the desired duration time whereas CP-DS and CP-CG gels with low adhesive property were easily flow out from tooth surface area and washed away by the surrounding medium. CPCG showed higher adhesive property than CP-DS. However, the drug released from CP-CG was lower than CP-DS. Therefore after the 4-h and 8-h regimens, the tooth whitening efficacy of CP-DS and CP-CG were quite similar and significantly lower than CP-HN.

In conclusion, the pigmented rice gels containing CP can be successively prepared using carboxymethyl modified rice as gelling agent. Different rice varieties yield the gels with different physicochemical properties leading to the difference tooth whitening efficacy of the gels. Moreover, the efficacy of CP rice gels is time dependent. For the 1-h regimen, CP-DS was the most effective tooth whitening gels whereas at the 4-h and 8-h regimen $\mathrm{CP}-\mathrm{HN}$ was the most effective tooth whitening gels.

\section{Acknowledgements}

The authors would like to thank the Thailand Research Fund for the financial support through the Research and Researcher for Industry (Grant No. PHD58I0012). We also thank the Agricultural Research Development Agency and the Higher Education Research Promotion 
and National Research University Project of Thailand, Office of the Higher Education Commission for their supports.

\section{References}

1. Dahl JE, Pallesen U. Tooth bleaching - A critical review of the biological aspects. Crit Rev Oral Biol Med. 2003; 14:292-304.

2. Sánchez AR, Rogers RS, Sheridan PJ. Tetracycline and other tetracycline-derivated staining of the teeth and oral cavity. Int J Dermatol. 2004; 43:709-715.

3. Hattab FN, Qudeimat MA, Al-Rimawi HS. Dental discoloration: An overview. J Esthet Restor Dent. 1999; 11:291-310.

4. Afroz S, Rathi S, Rajput G, Rahman SA. Dental esthetics and its impact on psycho-social well-being and dental self confidence: A campus based survey of north indian university students. J Indian Prosthodont Soc. 2013; 13:455-460.

5. Boeira GF, Salas MMS, Araújo DC, Masotti AS, Correa MB, Demarco FF. Factors influencing dental appearance satisfaction in adolescents: A cross-sectional study conducted in Southern Brazil. Brazilian J Oral Sci. 2016; 15:8-15.

6. Ibiyemi O, Taiwo J. Psychosocial aspect of anterior tooth discoloration among adolescents in igbo-ora, southwestern Nigeria. Ann Ib Postgrad Med. 2011; 9:9499.

7. Joiner A. The bleaching of teeth: A review of the literature. J Dent. 2006; 34:412-419.

8. Sulieman M. An overview of bleaching techniques: I. History, chemistry, safety and legal aspects. Dent Update. 2004; 31:608-610.

9. Abouassi T, Hann P, Wolkewitz M. Effect of carbamide peroxide and hidrogen peroxide on enamel surface: An in vitro study. Clin Oral Investig. 2011; 15:673-680.

10. Pinto CF, Oliveira R De, Cavalli V, Giannini M. Peroxide bleaching agent effects on enamel surface microhardness, roughness and morphology. Braz Oral Res. 2004; 18:306-311.

11. ADA Council on Scientific Affairs. Tooth whitening/ bleaching: Treatment considerations for dentists and their patients. American Dental Association, Chicago, IL, USA, 2009; pp. 1-12.

12. Chiu C, Solarek D. Modification of Starches. In: Starch: chemistry and technology (Be J, Whistler R, eds.). Third edition, Elsevier, 2009; pp. 629-655.

13. Glover DV, Mertz ET. Corn. In: Nutritional Quality of Cereal Grains: Genetic and Agronomic Improvement, Agronomy Monograph 28 (Olson RA, Frey KJ, eds.). American Society of Agronomy, Crop Science Society of America, Soil Science Society of America, Madison, WI, 1987; pp. 183-336.

14. Ramesh M, Bhattacharya KR, Mitchell JR. Developments in understanding the basis of cooked-rice texture. Crit Rev Food Sci Nutr. 2000; 40:449-460.

15. Okonogi S, Kaewpinta A, Yotsawimonwat S, Khongkhunthian S. Preparation and characterization of lidocaine rice gel for oral application. Drug Discov Ther. 2015; 9:397-403.

16. Okonogi S, Kaewpinta A, Khongkhunthian S, Yotsawimonwat S. Effect of rice variety on the physicochemical properties of the modified rice powders and their derived mucoadhesive gels. Drug Discov Ther. 2015; 9:221-228.

17. Okonogi S, Khongkhunthian S, Jaturasitha S. Development of mucoadhesive buccal films from rice for pharmaceutical delivery systems. Drug Discov Ther. 2014; 8:262-267.

18. Freire C, Podczeck F, Veiga F, Sousa J. Starchbased coatings for colon-specific delivery. Part II: Physicochemical properties and in vitro drug release from high amylose maize starch films. Eur J Pharm Biopharm. 2009; 72:587-594.

19. Junmahasathien T, Khongkhunthien S, Okonogi S. Preparation and characterization of modified colored rice as a gelling carrier for buccal drug delivery system. Int J Pharm Pharm Sci. 2016; 8:124-130.

20. Zaupa M, Calani L, Del Rio D, Brighenti F, Pellegrini N. Characterization of total antioxidant capacity and (poly)phenolic compounds of differently pigmented rice varieties and their changes during domestic cooking. Food Chem. 2015; 187:338-347.

21. Sumczynski D, Kotásková E, Družbíková H, Mlček J. Determination of contents and antioxidant activity of free and bound phenolics compounds and in vitro digestibility of commercial black and red rice (Oryza sativa L.) varieties. Food Chem. 2016; 211:339-346.

22. Yoenyongbuddhagal S, Noomhorm A. Effect of raw material preparation on rice vermicelli quality. Starch/ Staerke. 2002; 54:534-539.

23. Han RY, Fang JY, Sung KC, Hu OYP. Mucoadhesive buccal disks for novel nalbuphine prodrug controlled delivery: Effect of formulation variables on drug release and mucoadhesive performance. Int J Pharm. 1999; 177:201-209.

24. Deepthi V, Khan AB. Role of adhesives in transdermal drug delivery: A review. Int J Pharm Sci Res. 2012; 3:3559-3564.

25. Borges BCD, Borges JS, de Melo CD, Pinheiro IVA, dos Santos AJS, Braz R, Montes MAJR. Efficacy of a novel at-home bleaching technique with carbamide peroxides modified by CPP-ACP and its effect on the microhardness of bleached enamel. Oper Dent. 2011; 36:521-528.

26. De Jaime IML, França FMG, Basting RT, Turssi CP, Amaral FLB. Efficacy of hydrogen-peroxide-based mouthwash in altering enamel color. Am J Dent. 2014; 27:47-50.

27. Gimeno P, Bousquet C, Lassu N, Maggio AF, Civade C, Brenier C, Lempereur L. High-performance liquid chromatography method for the determination of hydrogen peroxide present or released in teeth bleaching kits and hair cosmetic products. J Pharm Biomed Anal. 2015; 107:386-393.

28. Pfeiffer K, Heinze T, Lazik W. Starch derivatives of high degree of functionalization 5. Stepwise carboxymethylation of amylose. Chem Pap. 2002; 56:261-266

29. Féliz-Matos L, Hernández LM, Abreu N. Dental bleaching techniques; hydrogen-carbamide peroxides and light sources for activation, an update. Open Dent J. 2014; 8:264-268.

30. Smart JD. The basics and underlying mechanisms of mucoadhesion. Adv Drug Deliv Rev. 2005; 57:15561568.

31. Oliveira GM, Miguez PA, Oliveira GB, Swift EJ, Farrell S, Anastasia MK, Conde E, Walter R. Safety and efficacy 
of a high-adhesion whitening strip under extended wear regimen. J Dent. 2013; 41:e46-52.

32. Anderson DG, Chiego DJ, Glickman GN, McCauley LK. A clinical assessment of the effects of $10 \%$ carbamide peroxide gel on human pulp tissue. J Endod. 1999; 25:247-250.

33. Walsh LJ. Safety issues relating to the use of hydrogen peroxide in dentistry. Aust Dent J. 2000; 45:257-269.
34. Haywood VB. A comparison of at-home and in-office bleaching. Dent Today. 2000; 19:1-5.

35. Haywood VB. Considerations for vital nightguard tooth bleaching with $10 \%$ carbamide peroxide after nearly 20 years of proven use. Inside Dent. 2006:2-5.

(Received May 7, 2018; Revised May 22, 2018; Accepted June 8, 2018) 